\title{
Del criterio cuantitativo al criterio cualitativo en la evaluación de impacto ambiental
}

\author{
From the quantitative to the qualitative criterion \\ in environmental impact assessment
}

\author{
KARIM KAHATT \& CECILIA AZERRAD*
}

Resumen: El ensayo analiza los criterios previstos en el régimen de evaluación de impacto ambiental peruano para determinar la obligatoriedad de la modificación de los estudios de impacto ambiental como consecuencia de la modificación de los proyectos. Identifica los vacíos existentes en la legislación transectorial y las deficiencias de los regímenes sectoriales, los que han privilegiado criterios de determinación fundamentalmente cuantitativos asociados a la magnitud del proyecto, sobre criterios cualitativos asociados al real impacto de la modificación.

Palabras clave: Sistema Nacional de Evaluación de Impacto Ambiental estudio de impacto ambiental - declaración de impacto ambiental - estudio de impacto ambiental semidetallado - certificación ambiental

Summary: The essay discusses the criteria available in Peru's environmental impact assessment regime for mandatory modification of environmental impact studies as a result of the modification of the underlying project. The paper identifies the gaps in the cross-sector legislation on environmental impact assessment, and the deficiencies of sector-specific legislation on environmental impact assessment, which have privileged quantitative criteria associated to the size of the project, over qualitative criteria associated to the real impact of the amendment of the project.

Key words: National System of Environmental Impact Assessment environmental impact study - environmental impact statement - semidetailed environmental impact study - environmental certification

CONTENIDO: I. INTRODUCCIÓN.- II. MARCO NORMATIVO AMBIENTAL GENERAL.- III. SISTEMA NACIONAL DE EVALUACIÓN DE IMPACTO AMBIENTAL.- IV. PRINCIPIO DE INDIVISIBILIDAD DE LA EVALUACIÓN DE IMPACTO AMBIENTAL.- V. MODIFICACIÓN DEL INSTRUMENTO DE EVALUACIÓN DE IMPACTO AMBIENTAL.- V.1. MODIFICACIÓN DEL EIA

* Karim Kahatt es socio a cargo del área ambiental de Santivañez Abogados, Lima, Perú. Abogado titulado por la Pontificia Universidad Católica del Perú, magíster en Derecho por The University of Texas at Austin, y magíster en Política Ambiental y Desarrollo por The London School of Economics and Political Science. Correo electrónico: Karim.kahatt@santivanez.com.pe

Cecilia Azerrad es asociada senior del área ambiental de Santivañez Abogados, Lima, Perú. Abogada titulada por la Pontificia Universidad Católica del Perú, magíster en Derecho por The University of Texas at Austin, y magíster en Derecho Ambiental y de la Sostenibilidad por la Universidad de Alicante. Correo electrónico: cecilia.azerrad@santivanez.com.pe 
EN EL REGLAMENTO DE PROTECCIÓN AMBIENTAL PARA ACTIVIDADES MINERO-METALÚRGICAS.- V.2. MODIFICACIÓN DEL EIA EN EL REGLAMENTO DE PROTECCIÓN AMBIENTAL PARA ACTIVIDADES ELÉCTRICAS.V.3. MODIFICACIÓN DEL EIA EN EL REGLAMENTO DE PROTECCIÓN AMBIENTAL PARA ACTIVIDADES DE HIDROCARBUROS.- VI. ENSAYO DE UNA INTERPRETACIÓN INTEGRADORA.- VI.1 MODIFICACIÓN DEL EIA.VI.2 MODIFICACIÓN DE LA DIA Y EL EIA-SD.- VII. CONCLUSIONES.

\section{INTRODUCCIÓN}

La legislación ambiental en el Perú ha tenido un significativo avance en los últimos años. El régimen legal ambiental seminal en toda legislación ambiental — la evaluación de impacto ambiental — se ha consolidado y fortalecido con mayor claridad desde septiembre de 2009, con la entrada en vigencia del reglamento de la Ley del Sistema Nacional de Evaluación de Impacto Ambiental ${ }^{1}$. A raíz de ello, la ley y el reglamento del Sistema Nacional de Evaluación de Impacto Ambiental, y con ellos, el Sistema Nacional de Evaluación de Impacto Ambiental (SEIA), han adquirido plena eficacia y aplicabilidad.

Desde la entrada en vigencia del reglamento del SEIA, el Sistema de Evaluación de Impacto Ambiental ha extendido su aplicación a un mayor número de proyectos y los ha clasificado en categorías ${ }^{3}$, de acuerdo con la significación de sus impactos. Asimismo, se han modificado las reglas o parámetros para la determinación de la obligatoriedad de la certificación ambiental y del contenido del mismo, quitándole protagonismo al criterio de magnitud de un proyecto, para adoptarse un criterio mixto de determinación. Criterio en el que, además de la magnitud del proyecto, se integra en el análisis, la determinación y el contenido del instrumento ambiental los impactos ambientales que el proyecto genera, en especial sobre los aspectos ambientales más sensibles.

Sin embargo, aun cuando el SEIA establece parámetros más coherentes en defensa del medio ambiente al integrarse en un contexto de certificación ambiental sectorial, mantiene varias ataduras a este paradigma cuantitativo, en función de la envergadura del proyecto, en lugar de priorizar el paradigma asociado a los verdaderos nuevos impactos generados por la modificación del proyecto subyacente. Precisamente,

1 Decreto supremo 019-2009-MINAM, que aprueba el reglamento de la Ley del Sistema Nacional de Evaluación de Impacto Ambiental.

2 Ley 27446, Ley del Sistema Nacional de Evaluación de Impacto Ambiental.

3 Antes de la entrada en vigencia del reglamento de la Ley del SEIA, en el subsector eléctrico, por ejemplo, únicamente se reconocía la figura del estudio de impacto ambiental (EIA) para aquellos proyectos eléctricos que requirieran contar con concesión definitiva, así como para la generación termoeléctrica con una potencia instalada mayor a 20MW. Luego de su entrada en vigencia, se ha ampliado la exigencia de contar con un instrumento de evaluación de impacto ambiental a aquellos proyectos que no requieran de concesión definitiva, o que requieran únicamente de autorización. 
estas ataduras a criterios rígidos y poco acertados, como la magnitud de un proyecto, están presentes en los regímenes sectoriales que forman parte del SEIA, mas no en las normas transectoriales.

Este ensayo reflexiona sobre cómo la estructura actual del sistema de evaluación de impacto ambiental aún deja de lado el paradigma cualitativo de determinación de impactos ambientales, centrándose en el modelo cuantitativo de envergadura del proyecto. La reflexión se hace a propósito del análisis de los criterios para determinar la obligatoriedad de la modificación de los estudios de impacto ambiental, y de la necesidad de uniformizarlos. Como contraparte a la identificación de los vacíos y deficiencias del régimen actual, se plantea una interpretación integradora que recoja los nuevos parámetros establecidos en el SEIA, los mismos que se basan en la determinación cualitativa de impactos sobre el medio ambiente.

Concretamente, se analiza la regulación existente sobre modificación de los distintos instrumentos de evaluación del impacto ambiental, por tratarse de un eslabón no resuelto por la Ley del SEIA y su reglamento. El ensayo ofrece una crítica sobre la regulación existente, con la finalidad de intentar una interpretación que integre la regulación ambiental en su conjunto, a fin de generar criterios que informan la obligatoriedad de la modificación de los instrumentos de evaluación ambiental para las principales actividades extractivas y energéticas que se desarrollan en el Perú.

Asimismo, se analizarán algunos avances que ha presentado la legislación ambiental subsectorial sobre modificación de proyectos y de los correspondientes instrumentos de evaluación ambiental. La misma que sobre la base de criterios cuantitativos integra algunos criterios ambientales cualitativos a la determinación de la obligatoriedad de la modificación de los instrumentos de evaluación ambiental. Sin embargo, se analizan también las limitaciones que presenta esta regulación en cuanto a la inclusión de dichos aspectos cualitativos.

\section{MARCO NORMATIVO AMBIENTAL GENERAL}

La Ley General del Ambiente, ley 28611, que aspira a ser la norma ordenadora del marco normativo legal para la gestión ambiental en el Perú, establece los principios y normas básicas para el ejercicio del derecho constitucional al ambiente saludable, equilibrado y adecuado para el pleno desarrollo de la vida. Asimismo, regula el cumplimiento de las obligaciones vinculadas a la efectiva gestión ambiental: que implique la mejora de la calidad de vida de la población, el desarrollo sostenible de las actividades económicas, el aprovechamiento sostenible de los recursos naturales, el mejoramiento del ambiente urbano y rural,

DEL CRITERIO CUANTITATIVO AL CRITERIO CUALITATIVO EN LA EVALUACIÓN DE IMPACTO AMBIENTAL

FROM THE

QUANTITATIVE TO THE QUALITATIVE CRITERION IN ENVIRONMENTAL IMPACT

ASSESSMENT 
así como la conservación del patrimonio natural del país, entre otros objetivos.

La Ley General del Ambiente, entre otras normas, establece la obligatoriedad de la presentación de un instrumento de evaluación de impacto ambiental para todos los proyectos de obra o actividad de carácter público o privado que puedan provocar daños intolerables al medio ambiente.

En ese sentido, establece en el inciso 1 del artículo 24, que:

[...] toda actividad humana que implique construcciones, obras, servicios y otras actividades, así como las políticas, planes y programas públicos susceptibles de causar impactos ambientales de carácter significativo, está sujeta, de acuerdo a la ley, al Sistema Nacional de Evaluación del Impacto Ambiental - SEIA, el cual es administrado por la Autoridad Ambiental Nacional.

Sin embargo, la Ley General del Ambiente carece de contenido normativo propio, limitándose a efectuar una remisión en blanco a lo que disponga la Ley del SEIA, su reglamento y las demás normas sobre la materia. Ello no hubiese tenido mayor relevancia, pues desde 2001 existe la Ley del SEIA. Sin embargo, la Ley del SEIA tampoco determina por sí misma el ámbito de aplicación del sistema. Es decir, las actividades o proyectos, públicos o privados, que deben ser sometidos a evaluación de impacto ambiental, con carácter previo a su aprobación o autorización. Como se conoce, esta determinación del ámbito de aplicación, un aspecto tan esencial del régimen jurídico ambiental, fue remitida al reglamento de la Ley de SEIA, el cual tuvo que esperar ocho años desde la promulgación de dicha ley para su aprobación. Para salvar esta dificultad, la propia ley disponía que serían de aplicación las normas sectoriales sobre la materia en la medida en que no se opusieran a la Ley del SEIA.

Entonces, si bien existía una obligatoriedad de obtener una «certificación ambiental» (autorización administrativa) para poder iniciar la ejecución de proyectos con impacto ambiental significativo, los proyectos comprendidos en el SEIA y las características de la propia evaluación se han mantenido en manos de cada sector de actividad, habiéndose adoptado la regulación transectorial de manera supletoria para aquellos proyectos que no se encuentran contemplados en cada regulación sectorial de protección ambiental. 


\section{SISTEMA NACIONAL}

La ley 27446, Ley del Sistema Nacional de Evaluación de Impacto Ambiental y su respectivo reglamento (decreto supremo 019-2009. MINAM) establecen el régimen transectorial de certificación ambiental para todos los proyectos que se encuentren dentro del Sistema Nacional de Evaluación del Impacto Ambiental. Los proyectos contenidos dentro del SEIA se encuentran establecidos en el anexo II del reglamento de la Ley del SEIA.

En ese sentido, de acuerdo con el artículo 4 de la ley 27446, Ley del SEIA, toda actividad respecto de la cual se solicite su certificación ambiental deberá ser clasificada en una de las siguientes categorías:

Categoría I. Declaración de impacto ambiental. Incluye aquellos proyectos cuya ejecución no origina impactos ambientales negativos de carácter significativo.

Categoría II. Estudio de impacto ambiental semidetallado. Incluye los proyectos cuya ejecución puede originar impactos ambientales moderados y cuyos efectos negativos pueden ser eliminados o minimizados mediante la adopción de medidas fácilmente aplicables.

Categoría III. Estudio de impacto ambiental detallado para proyectos de mayor envergadura y potenciales impactos negativos.

En el caso de las actividades mineras, la Ley del SEIA y su reglamento son solo de aplicación supletoria, en tanto las actividades minero metalúrgicas, como las actividades de exploración minera, cuentan con sus propios reglamentos de protección ambiental que regulan la certificación ambiental para proyectos mineros. Más adelante se analizan estos subregímenes en cuanto a evaluación de impacto ambiental se refiere.

Lo mismo ocurre con el subsector de hidrocarburos. El subsector de hidrocarburos cuenta con el reglamento de protección ambiental para las actividades de hidrocarburos, aprobado por decreto supremo 015-2006-EM. Y también ocurre algo similar, aunque en menor medida, con el sector eléctrico que cuenta con su reglamento sectorial de protección ambiental de las actividades eléctricas, aprobado por decreto supremo 29-94-EM. Se afirma que la situación es similar, aunque en menor medida, porque la aplicación supletoria en el sector eléctrico es mucho más necesaria que en los otros dos subsectores. Esto se debe a que el subsector eléctrico no ha adecuado su régimen legal de evaluación de impacto ambiental al régimen transectorial. El régimen legal ambiental eléctrico regula la exigencia del estudio de impacto ambiental («EIA a secas»), como instrumento propio del subsector, para

DEL CRITERIO

CUANTITATIVO

AL CRITERIO

CUALITATIVO EN

LA EVALUACIÓN

DE IMPACTO

AMBIENTAL

FROM THE

QUANTITATIVE TO

THE QUALITATIVE

CRITERION IN

ENVIRONMENTAL

IMPACT

ASSESSMENT 
aquellas actividades eléctricas que requieran de concesión definitiva, pero no contempla instrumentos de evaluación de impacto ambiental de menor complejidad para actividades que no requieren concesión definitiva, o que requiriendo, tienen menores impactos ambientales que los de un proyecto que por su magnitud y sus impactos amerita la preparación de un estudio de impacto ambiental (EIA). Más adelante se ofrece un análisis más detallado de estos regímenes legales de evaluación de impacto ambiental.

Ahora bien, cuando un proyecto se encuentra dentro del anexo II del reglamento del SEIA, el titular del proyecto deberá seguir el procedimiento de clasificación de los proyectos de inversión señalado en los artículos 6, 7 y 8 de la Ley del SEIA, y 36 y siguientes del reglamento. La finalidad de este procedimiento es que sobre la base de una evaluación preliminar se clasifique dicho proyecto en una de las tres categorías establecidas en el artículo 4 de la Ley del SEIA.

La riqueza que introduce este sistema es que los criterios para determinar la categoría en la cual se encuentra cada uno de los proyectos sometidos a certificación ambiental se establece en función de los potenciales impactos que generaría la actividad. En ese sentido, el anexo $\mathrm{V}$ del reglamento de la Ley del SEIA establece como criterios para la determinación del tipo de instrumento ambiental los siguientes:

- la protección de la salud pública y de las personas;

- la protección de la calidad ambiental, tanto del aire, del agua, del suelo, como la incidencia que puedan producir el ruido y vibración, residuos sólidos y líquidos, efluentes, emisiones gaseosas, radiaciones y de partículas y residuos radiactivos;

- la protección de los recursos naturales, especialmente las aguas, los bosques y el suelo, la flora y fauna;

- la protección de las áreas naturales protegidas;

- la protección de la diversidad biológica y sus componentes: ecosistemas, especies y genes; así como los bienes y servicios ambientales y bellezas escénicas, áreas que son centros de origen y diversificación genética por su importancia para la vida natural;

- la protección de los sistemas y estilos de vida de las comunidades campesinas, nativas y pueblos indígenas;

- la protección de los espacios urbanos; y

- la protección del patrimonio arqueológico, histórico, arquitectónico y monumentos nacionales.

Esto quiere decir que el reglamento de la Ley del SEIA deja de lado la clasificación cuantitativa sobre la base de la envergadura del proyecto para centrarse en una clasificación sobre la base de criterios ambientales. 
Por ello, para determinar la categoría a la que pertenece un proyecto específico, deberá analizarse el impacto potencial del mismo sobre el medio ambiente, tomando en consideración los criterios mencionados.

\section{PRINCIPIO DE INDIVISIBILIDAD DE EVALUACIÓN DE IMPACTO AMBIENTAL}

DEL CRITERIO

CUANTITATIVO

AL CRITERIO

El estudio de impacto ambiental (EIA) es aquel instrumento de gestión ambiental que busca identificar cuáles serán los posibles impactos que genere el desarrollo de una determinada actividad, tratando de minimizar o evitar los impactos negativos significativos y potenciar aquellos impactos positivos.

El autor Juan Rosa Moreno menciona que los EIA están conformados por elementos subjetivos y objetivos; y dentro de estos últimos encontramos los principios de proporcionalidad, rigurosidad y universalidad. Con respecto al principio de universalidad, señala que:

Este principio material hace referencia a la tipología de componentes y de impactos a analizar, es decir, es relativo al horizonte material de los estudios de impacto. [...] La universalidad también se imputa a los diversos tipos de efectos que deben analizarse. [...] El Real Decreto 1131/1.988 cumpliendo, quizá en exceso, su inherente función de desarrollo, impone el deber de analizar, distinguiéndolos, los efectos positivos de los negativos; los temporales de los permanentes; los simples de los acumulativos y sinérgicos; los reversibles de los irreversibles; los recuperables de los irrecuperables; los periódicos de los de aparición irregular; los continuos de los discontinuos; y los directos de los indirectos'.

Entonces, el EIA busca determinar cuáles serán los componentes e impactos a analizar, derivados de la actividad humana y qué medidas se adoptarán para mitigar aquellos negativos y potenciar los positivos. Es en función de los impactos que se producirán que se hace imprescindible realizar una evaluación conjunta de todos los componentes que conforman el proyecto así como los impactos que se generarán, considerando que se pueden generar impactos acumulativos. Estos pueden ser resultado de actuaciones de menor importancia vistas individualmente, pero significativas en su conjunto.

En ese mismo sentido, el autor José Francisco Alenza García menciona que:

4 Rosa Moreno, Juan. Régimen jurídico de la evaluación de impacto ambiental. Madrid: Trivium, 1993, p. 224.

CUALITATIVO EN

LA EVALUACIÓN

DE IMPACTO

AMBIENTAL

FROM THE

QUANTITATIVE TO

THE QUALITATIVE

CRITERION IN

ENVIRONMENTAL

IMPACT

ASSESSMENT 
En la STJCE de 21 de septiembre de 1999, Comisión contra Irlanda (C-392/96) se dijo lo siguiente: «Así, un Estado miembro que estableciera los criterios y/o umbrales en un nivel tal que, en la práctica, la totalidad de los proyectos de un determinado tipo quedara a priori exenta de la obligación del estudio sobre impacto ambiental sobrepasaría el margen de apreciación de que dispone en virtud del artículo 2, apartado 1, y del artículo 4, apartado 2 de la Directiva, salvo que, sobre la base de una apreciación global, pudiera considerarse que ninguno de los proyectos excluidos podía tener repercusiones importantes sobre el medio ambiente.

Así sucedería en el supuesto de un Estado miembro que se limitase a fijar un criterio de dimensión de los proyectos y no se asegurase por otra parte, de que no se eludiera el objetivo de la normativa mediante un fraccionamiento de los proyectos. En efecto, el hecho de que no se tenga en cuenta el efecto acumulativo de los proyectos tiene como consecuencia práctica que la totalidad de los proyectos de un determinado tipo puede quedar exenta de la obligación de evaluación, aunque considerados conjuntamente, pueden tener repercusiones importantes sobre el medio ambiente en el sentido del artículo 2, apartado 1, de la Directiva» ${ }^{5}$.

Mediante esta sentencia se reafirma el principio de indivisibilidad, mediante el cual se determina que es necesario tomar en cuenta los efectos acumulativos que, de ser considerados individualmente, podrían pasar inadvertidos, pero que si fueran considerados de manera conjunta, podrían tener impactos significativos sobre el medio ambiente.

Este principio de indivisibilidad está reconocido en el reglamento de la Ley del SEIA como aquel que establece la necesidad de recoger dentro del instrumento de evaluación de impacto ambiental, de manera indivisa, todos los componentes del proyecto; así como las medidas y acciones concretas, viables y de obligatorio cumplimiento para asegurar de manera permanente el adecuado manejo ambiental de dichos componentes, así como un buen desempeño ambiental en todas sus fases. Como aplicaciones directas de este principio, se puede concluir que queda prohibido, por ejemplo: dividir artificialmente un mismo proyecto en dos proyectos similares, contiguos o cercanos, de menor envergadura e impactos, para simular una menor afectación y merecer una clasificación menor a la que le hubiera correspondido, y un estudio de menores exigencias, en caso de haber sido descrito en su conjunto; dividir artificialmente un proyecto en la construcción

5 AlenZa García, José Francisco. «La evaluación de impacto ambiental a la luz de la jurisprudencia comunitaria». En NOGUEIRA LÓPEZ, Alba (coord.). Evaluación de impacto ambiental: Evolución normativo-jurisprudencial, cuestiones procedimentales y aplicación sectorial. Barcelona: Atelier, 2009, p. 45. 
de la infraestructura y la implementación de la actividad; entre otras estrategias que buscan reducir el nivel de exigencia para los proyectos.

\section{MODIFICACIÓN DEL INSTRUMENTO DE EVALUACIÓN DE IMPACTO AMBIENTAL}

En lo que queda de este artículo, se analizará concretamente la regulación existente sobre modificación de los distintos instrumentos de evaluación del impacto ambiental, por tratarse de un eslabón no resuelto por la Ley del SEIA y su reglamento. En ese sentido, se presentará de manera crítica la regulación existente con la finalidad de intentar una interpretación que integre la regulación ambiental en su conjunto. Esto permitiría, a su vez, llenar de contenido las reglas o parámetros que informan la modificación de los instrumentos de evaluación ambiental en el sector minero, en el sector eléctrico y en el de hidrocarburos.

El régimen transectorial no ofrece parámetros o indicadores específicos que permitan determinar la posibilidad de modificar el diseño original de un proyecto sobre el cual ha sido aprobado el instrumento de evaluación de impacto ambiental. De acuerdo con el artículo 58 del reglamento de la Ley del SEIA, la autoridad competente deberá aprobar las normas que regulen las condiciones y procedimientos para la modificación, suspensión y cancelación de la certificación ambiental.

Esto significa que los criterios para la modificación de los EIA, al día de hoy y mientras la institucionalidad ambiental se encuentre diseminada, se encuentran en la regulación sectorial. Por lo tanto, corresponde analizar si dicha salida favorece el control ambiental o si, por el contrario, es rígida y desligada de los principios que informan la normativa ambiental instaurada por las normas del SEIA, vigentes desde el año 2009. El análisis será realizado a partir de la normativa sectorial minera, eléctrica y de hidrocarburos, destacando los supuestos más saltantes sobre la necesidad de implementar la modificación de proyectos y la correspondiente modificación de los respectivos instrumentos de evaluación de impacto ambiental.

\section{V.1. Modificación del ElA en el reglamento de protección ambiental para actividades minero-metalúrgicas}

El reglamento del Título Décimo Quinto del Texto Único Ordenado de la Ley General de Minería, sobre el medio ambiente, aprobado por decreto supremo 016-93-EM, establece las normas sobre certificación ambiental para las actividades minero metalúrgicas. El inciso 3 del artículo 7 del reglamento, modificado por la Primera Disposición Complementaria Final del decreto supremo 078-2009-EM, publicado el 8 noviembre de 2009, establece lo siguiente: «Los titulares mineros

DEL CRITERIO CUANTITATIVO AL CRITERIO

CUALITATIVO EN LA EVALUACIÓN DE IMPACTO AMBIENTAL

FROM THE

QUANTITATIVE TO THE QUALITATIVE CRITERION IN ENVIRONMENTAL IMPACT

ASSESSMENT 
que se encuentren en la etapa de explotación y que requieren ampliar el volumen de sus operaciones extractivas, deberán presentar ante el Ministerio de Energía y Minas la modificación del Estudio de Impacto Ambiental aprobado para tal actividad».

Por su parte, el artículo 20 señala que el concesionario minero o de beneficio que proyecte realizar ampliaciones de producción en sus operaciones o de tamaño de planta de beneficio superior al cincuenta por ciento se deberá sujetar a lo dispuesto en la parte final del inciso 3 del artículo 7 del presente reglamento, debiendo presentar un nuevo EIA. El artículo 20 también señala excepciones concretas a la necesidad de modificar formalmente el EIA, cuando la ampliación se encuentre en alguno de los supuestos indicados a continuación:

a) Ampliaciones de producción en sus operaciones sin afectar nuevas áreas o exceder los límites de las áreas que fueron determinadas y evaluadas en el EIA o PAMA de la concesión de beneficio vigente.

b) En el caso de recrecimiento de relaveras, pads de lixiviación y desmonteras, cuando el recrecimiento o ampliación de estos componentes no afecte nuevas áreas o no exceda los límites de las áreas que fueron determinadas y evaluadas para dichos componentes en el EIA o PAMA que los consideró.

c) Cuando se trate de mejoras tecnológicas en la planta o sustitución de equipos, siempre que no implique un mayor consumo de agua o nuevas áreas no consideradas en el EIA o PAMA.

La primera crítica que puede realizarse respecto de los artículos que regulan la modificación del instrumento de evaluación de impacto ambiental, que para las actividades minero-metalúrgicas corresponde a un EIA, es que únicamente se centra en la ampliación cuantitativa de las operaciones. Es decir, el criterio para determinar si un EIA requiere de una modificación con aprobación formal por parte de la autoridad competente se realiza sobre la base estricta de la expansión de las operaciones en un cincuenta por ciento y no sobre los componentes ambientales que podría estar afectando.

Se debe reconocer que las excepciones a la regla descritas precedentemente establecen también un criterio objetivo acertado: se requiere someter a aprobación de la autoridad la modificación de un EIA que implique áreas nuevas. Este criterio es difícil de clasificar como cuantitativo o como cualitativo. Es probable que sea un poco de ambos. Cuantitativo porque se refiere a magnitudes (más área afectada). Cualitativo porque se refiere a tipos de impacto (nueva área afectada, con una línea base independiente). Más allá de la clasificación, este criterio es adecuado y coherente, dado que sigue la 
naturaleza inherente a la evaluación de impacto ambiental (donde se va a desarrollar un proyecto debe medirse la línea base, prever los impactos y señalar su forma de mitigación). Sin embargo, no es suficiente. Debe ser empleado como uno entre varios criterios de contenido ambiental. Tampoco puede ser categórico. Podría tratarse de una minúscula porción de área nueva con casi idénticas características al área ya recogida en el estudio de línea base.

La Ley del SEIA y su reglamento han aprovechado la oportunidad para introducir criterios cualitativos de determinación sobre la base de la potencial afectación del medio ambiente, trasladando al titular minero la responsabilidad de fijar los criterios. No obstante, la autoridad sectorial minera ha mantenido el criterio original de determinación cuantitativa para determinar la necesidad de aprobación de una modificación del EIA. El acercamiento más cercano a la determinación sobre la base de criterios ambientales de la modificación formal de un EIA lo encontramos en el inciso (c) del artículo 20, el cual establece que la ampliación de las instalaciones no implique mayor consumo de agua. Sin embargo, este es un tímido intento por incluir componentes ambientales, que no llega a reflejar a las nuevas tendencias que ofrece la legislación actual sobre medio ambiente, y la política general medioambiental, recogida en el anexo $\mathrm{V}$ del reglamento de la Ley del SEIA.

El segundo defecto que presenta la norma analizada es que no distingue claramente los supuestos que ameritan una modificación del EIA de aquellos que dan origen a un nuevo EIA. Ello sucede porque en el momento en que entró en vigencia el decreto supremo 016-93-EM, en el año 1993, se pretendía regular a aquellas actividades ya existentes que no contaban con ningún instrumento de gestión ambiental. En ese sentido, dicho reglamento introdujo el Programa de Adecuación y Manejo Ambiental (PAMA) para proyectos existentes y reguló los supuestos en los cuales las nuevas actividades requerirían de EIA.

Dicha realidad hoy en día ha cambiado, haciendo menos probable que una actividad sujeta a certificación ambiental no cuente con ella. Por ello, en virtud del principio de indivisibilidad que acompaña a los instrumentos de evaluación de impacto ambiental de cada una de las distintas categorías, los impactos ambientales de un mismo proyecto deben ser contemplados acumulativamente en un solo instrumento, con la finalidad de enfrentar sus efectos de manera integral. En ese sentido, en la actualidad, aun cuando la norma señale el requisito de elaborar un nuevo EIA, deberá entenderse que se trata de la modificación de aquellos componentes del EIA que se verían afectados por la ampliación del proyecto, así como sus medidas de manejo ambiental, tomando en consideración los impactos negativos que genera la actividad ampliada en su conjunto. Como resulta evidente, una vez que un área ha sido 
impactada, nunca más podremos referirnos a una nueva línea base, y por tanto, no resulta posible hablar de un «nuevo EIA». Cuando se trata de operaciones en marcha con un EIA aprobado que requiere modificación (con o sin aprobación de la modificación por parte de la autoridad) como consecuencia de la modificación del proyecto subyacente, siempre deberá partirse del EIA aprobado.

Otro de los problemas que presentan las normas sobre modificación o ampliación de los proyectos mineros es que la misma se limita a la las actividades mineras en sí mismas. Es decir, lo hace en relación con las actividades mineras propiamente dichas, sin considerar los cambios que puedan producirse en instalaciones o actividades conexas, como por ejemplo las actividades de generación y/o distribución de energía sin carácter de servicio público. Actividades estas que no solo tienen un marco legal diferente, sino que incluso su modificación está sujeta a la opinión previa favorable de otra autoridad distinta de la ambiental minera.

La evaluación del impacto ambiental se realiza de manera integral e integrada sobre políticas, planes, programas y proyectos de inversión, comprendiendo de manera indivisa todos los componentes de los mismos. Asimismo, implica la determinación de medidas y acciones concretas, viables y de obligatorio cumplimiento para asegurar de manera permanente el adecuado manejo ambiental de dichos componentes, así como un buen desempeño ambiental en todas sus fases. En ese sentido, el EIA y su modificación deben incluir no solo la actividad minera en sí misma, sino los demás componentes que integran el proyecto aun de manera complementaria. La norma analizada, por tanto, no contempla dicho supuesto, dejando de lado a aquellas modificaciones o ampliaciones que escapan de la actividad principal y por tanto, del objetivo central del EIA.

El proyecto de reglamento de Gestión Ambiental para las Actividades de Explotación, Beneficio, Transporte y Almacenamiento Minero (que reemplazaría al decreto supremo 016-93-EM) contiene disposiciones innovadoras en relación con los criterios para determinar la obligatoriedad de la aprobación de la modificación de los EIA. Este proyecto, prepublicado en 2011, introduce criterios cualitativos a la determinación de la necesidad de modificación de un estudio de impacto ambiental detallado (EIAd).

Esta norma señala que las modificaciones o ampliaciones de los proyectos mineros, «que pudiesen generar nuevos o mayores impactos ambientales o sociales negativos, deberán ser aprobados previamente», a través de un procedimiento administrativo de modificación del EIAd. Por su parte, el mismo proyecto establece como excepción a la tramitación de la modificación de un EIAd que la modificación o ampliación de las 
actividades mineras implique la realización de actividades que «valoradas en conjunto con el EIAd inicial y sus modificaciones subsiguientes aprobadas, signifiquen un similar o menor impacto ambiental potencial». En ese sentido, este proyecto de reglamento para actividades mineras introduce claramente en el análisis el criterio cualitativo de valoración de impactos para la determinación de la necesidad de aprobación de la modificación de un instrumento de evaluación de impacto ambiental, dejando de lado el criterio cuantitativo que valora la magnitud del crecimiento o modificación de la actividad, en lugar de valorar, en concordancia con las normas del SEIA, la magnitud del potencial impacto ambiental que tal modificación originaría.

\section{V.2. Modificación del EIA en el reglamento de protección ambiental para actividades eléctricas}

El segundo subsector que conviene analizar es el eléctrico, cuyo régimen de evaluación de impacto ambiental de las actividades de generación, transmisión y distribución está contenido en el reglamento de Protección Ambiental para Actividades Eléctricas, aprobado por decreto supremo 029-94-EM. El artículo 20 de esta norma señala que:

Los sistemas eléctricos que se encuentren en operación, deberán presentar un EIA, para los casos en los que se considere una ampliación de sus instalaciones en más del cincuenta por ciento de su capacidad instalada y/o un incremento en un veinticinco por ciento de su nivel actual de emisiones y/o que involucre la utilización de nuevas áreas.

La remisión a los reglamentos ambientales sectoriales realizada por el artículo 58 del reglamento del SEIA puede dar lugar a interpretar que para las actividades eléctricas únicamente se encuentre regulada la modificación de aquellos proyectos iniciados antes de la entrada en vigencia de la norma en 1994. Es decir, para aquellos proyectos a los que se les exigía un PAMA a la entrada en vigencia de la norma; y para los cuales se contempló que, en caso de modificación o ampliación posterior, se requeriría de la elaboración y aprobación de un nuevo EIA.

Sin embargo, corresponde analizar si es posible interpretar por analogía que estos mismos criterios sean empleados para determinar la obligatoriedad de solicitar la aprobación de la modificación del EIA en casos de proyectos que cuenta con un EIA aprobado, ya sea que los mismos se encuentren operando, o para aquellos cuya infraestructura aún no haya sido construida, pero que se encuentra en proceso de construcción. De no ser así, la gran mayoría de supuestos de modificación de actividades eléctricas que se desarrollan en la actualidad no tendrían reglas para la modificación de su instrumento de evaluación de impacto ambiental. 
Por otro lado, la deficiente redacción de la norma induce a interpretar que cuando un proyecto se amplía en un cincuenta por ciento en relación con el proyecto inicial, se requiera la utilización de nuevas áreas, o se incrementen las emisiones en veinticinco por ciento se requerirá de un nuevo EIA.

Como se ha discutido precedentemente, no existe tal cosa como «un nuevo EIA» en los casos en los que el área del proyecto haya sido afectada, ya que nunca más se podrá contar con una nueva línea basea ambiental. Lo que corresponde claramente es la modificación del estudio existente, debido a que deberán integrarse en un solo instrumento todos los impactos acumulativos del proyecto general, los cuales deben manejarse de forma integrada.

Por último, la ausencia de regulación adicional al artículo 20 del reglamento de Protección Ambiental en las Actividades Eléctricas, determina que solo aquellas actividades que requieran de EIA cuenten con una regla que permite la modificación de los mismos. Para el caso de las actividades eléctricas, solo requieren de EIA aquellas actividades que para su desarrollo necesitan obtener una concesión definitiva, y, la generación térmica y la generación de recurso energéticos renovables con una potencia instalada mayor a $20 \mathrm{MW}$.

Las demás actividades eléctricas que no requieren contar con EIA aprobado de acuerdo con el reglamento sectorial de protección ambiental - ya sea porque no requieren de concesión o porque los proyectos no alcanzan la magnitud o los criterios que la norma impone como hito a partir del cual el EIA es exigible - requieren, a partir de la entrada en vigencia del reglamento de la Ley del SEIA, de certificación ambiental en virtud de las normas transectoriales, y por tanto, requieren de una declaración de impacto ambiental (DIA) o en su defecto de un EIA semidetallado (EIA-SD). A estos proyectos con estudios ambientales distintos a un EIA, entendido como la versión más compleja de los estudio ambientales, se les aplica el régimen de evaluación de impacto ambiental transectorial y se les seguirá aplicando dicho régimen hasta que se adecue el régimen sectorial de certificación ambiental del subsector eléctrico. Como se ha mencionado, este régimen ofrece pocos criterios para determinar la obligatoriedad de la modificación de los estudios ambientales.

\section{V.3. Modificación del ElA en el reglamento de protección ambiental para actividades de hidrocarburos}

A juzgar por los criterios que informan la obligatoriedad de la aprobación de la modificación de los estudios ambientales como consecuencia de la modificación de los proyectos subyacentes, este subsector es el 
más avanzado de los tres que integran el sector energía y minas. Esta situación se explica por la oportunidad de su aprobación. Mientras que los reglamentos ambientales sectoriales minero (explotación, beneficio, transporte y almacenamiento) y eléctrico (todas las actividades eléctricas) datan de inicios de los años noventa, y no han sido adecuados al régimen transectorial del SEIA, el reglamento sectorial de hidrocarburos data del año 2006 y está adecuado en gran medida al régimen transectorial de certificación ambiental. Se afirma que este es el más avanzado de los tres subsectores porque ha dejado de lado los criterios cuantitativos y ha adoptado criterios cualitativos para determinar en qué supuestos es obligatorio solicitar la aprobación de la modificación de los estudios ambientales como consecuencia de la modificación de los proyectos subyacentes.

Al respecto, el artículo 36 del reglamento de Protección Ambiental para las Actividades de Hidrocarburos, aprobado por decreto supremo 015-2006-EM, señala que el programa de manejo ambiental (PMA) deberá ser actualizado cuando el titular de la actividad de hidrocarburos considere necesario modificar las técnicas o procedimientos aprobados, o cuando el proceso productivo sufra modificaciones que impacten de manera diferente al ambiente físico y social, con relación a los impactos evaluados en los instrumentos de gestión ambiental.

A continuación, el mismo artículo dispone que dicha actualización deberá ser presentada a la autoridad competente, la Dirección General de Asuntos Ambientales Energéticos (DGAAE), y detalla los requisitos y el procedimiento de evaluación, pero no señala expresamente que dicha actualización debe ser aprobada por la autoridad. No obstante, la redacción del tercer párrafo de dicho artículo nos permite concluir que en esos casos (nótese que se refiere expresamente a las modificaciones que generen impactos distintos a los evaluados en los estudios aprobados) sí se exige una aprobación expresa de la autoridad competente.

A contrario sensu, podemos concluir que este reglamento ha dispuesto que si las modificaciones no impactan de manera distinta al ambiente físico y social, con relación a los impactos evaluados en los estudios aprobados, la modificación de la actividad no estará sujeta a aprobación de la autoridad competente. Lo que no llegó a señalar esta norma del reglamento, y que en todo caso constituye un vacío de esta norma, es la obligación de comunicar la modificación a la autoridad competente de otorgar la certificación ambiental; y a la autoridad competente de la fiscalización ambiental, la modificación del estudio para efectos de fiscalización.

Este reglamento contiene también un mecanismo complementario referido a modificaciones de los proyectos que generan cambios en los respectivos EIA. Está contenido en el artículo 62 y se le puede denominar 
«sistema de control de cambios». En virtud de esta herramienta, es el propio titular quien establece un mecanismo para llevar el control de los cambios que introduce en las instalaciones, los procesos, los procedimientos de operación, los procedimientos de mantenimiento, los procedimientos logísticos u otras actividades, para identificar y evaluar los efectos sobre la salud, la seguridad y el ambiente antes de implementar la modificación.

Esta norma no ofrece un criterio objetivo. Es más, resulta una disposición bastante vaga; más aun si consideramos la parte final del artículo que indica que «la implementación de la modificación podría requerir a su vez, modificar el PMA». Esta última parte de la disposición permite inferir que no todo cambio en las instalaciones y procesos de la actividad generan la obligación de modificar el PMA, menos aun de solicitar su aprobación por parte de la autoridad. Puede afirmarse que con una disposición como esta, queda claro que no todas las modificaciones requieren de aprobación y que incluso algunas de ellas pueden resultar tan específicas y limitadas que ni siquiera generarían la necesidad de modificar el estudio.

Al respecto, recordemos que la evaluación de impacto ambiental no es un listado que autoriza componentes o actividades, sino que es una herramienta que valida las medidas de gestión ambiental que se implementarán para evitar, mitigar o compensar los impactos generados por dichos componentes o autoridades. Por tanto, no existe ni puede crearse por interpretación analógica un criterio que pretenda asimilar los estudios ambientales a listados exhaustivos de actividades o componentes autorizados, cuya ausencia en el estudio los devenga prohibidos.

El proyecto de reglamento que reemplazará al reglamento vigente aprobado por decreto supremo 015-2006-EM corrige las pequeñas deficiencias y conserva los aciertos identificados en la norma vigente: i) se confirma que únicamente las modificaciones o ampliaciones del proyecto de hidrocarburos aprobados en un estudio ambiental, que pudiesen generar nuevos o mayores impactos ambientales, deben ser aprobadas previamente; ii) establece un procedimiento claro para la aprobación de la modificación propuesta; iii) dispone expresamente el requisito de participación ciudadana para aquellas modificaciones que requieran aprobación, ausente en otros regímenes de modificación de estudios ambientales; iv) prevé expresamente que las modificaciones o ampliación de actividades propuestas se desarrollen en el área de influencia directa, representen similar o menor impacto y no generen impactos nuevos a los identificados en el estudio ambiental aprobado no requieren de aprobación, sino tan solo de comunicación a la autoridad; y v) establece el requisito de informar a la población del área de influencia 
directa sobre los alcances de la modificación para aquellos casos que no requieran de la aprobación de la modificación. Un acierto.

\section{ENSAYO DE UNA INTERPRETACIÓN INTEGRADORA}

Como ha podido observarse, los reglamentos sectoriales de protección ambiental analizados no establecen con claridad los criterios para la modificación de cada una de las distintas modalidades de instrumento de evaluación de impacto ambiental. Dichos reglamentos tampoco se ciñen a los parámetros recogidos por las normas del SEIA. Sin embargo, a partir de los parámetros establecidos por las normas sectoriales y transectoriales existentes, se puede arribar a ciertas aproximaciones que si bien no surgen directamente de las normas sectoriales, responden a los principios que informan la actual normativa ambiental en su conjunto.

\section{VI.1. Modificación del EIA}

El EIA constituye un instrumento de gestión de impactos ambientales «significativos» que acompaña el desarrollo y operación durante toda la vida de un proyecto, ya sea minero, energético o de cualquier otra naturaleza. Por lo tanto, solo debería requerirse la aprobación formal, por parte de la autoridad ambiental competente, de una modificación del EIA ${ }^{6}$ cuando la actividad que fue materia de certificación ambiental varía sustancialmente.

En ese sentido, la clasificación de una modificación a un proyecto como susceptible de generar impactos significativos sobre el medio ambiente no solo debe tomar en cuenta la magnitud (cuantitativa) de tal variación, sino además debería requerirse de la modificación del EIA. Modificación que cuente con la aprobación previa de la autoridad ambiental competente, en la medida en que la nueva área a utilizar reporte características bióticas y ecológicas distintas a las previstas en el EIA en el cual se había concebido originalmente el proyecto, o cuando alguno de los ocho criterios recogidos en el anexo V del reglamento de la Ley del SEIA se vean alterados por la modificación o ampliación de la actividad respectiva, requiriéndose un nuevo pronunciamiento por parte de la autoridad.

Desde esta perspectiva, en la medida en que el proyecto se ubique en la misma área que la originalmente prevista en el EIA, y mantenga características bióticas y ecológicas comunes a las originales, no se debería exigir la aprobación previa de la modificación del EIA por parte

6 Como se ha sostenido a lo largo de este artículo, la modificación del instrumento de evaluación ambiental existente en lugar de la elaboración de un instrumento nuevo de evaluación de impacto ambiental se sustenta en el inciso a) del artículo 3 del reglamento de la Ley del SEIA, que recoge el principio de indivisibilidad, y el mismo que establece que deberán integrarse en un solo instrumento todos los impactos acumulativos del proyecto general, los cuales deben manejarse de forma integrada, comprendiendo de manera indivisa todos los componentes del proyecto.

DEL CRITERIO CUANTITATIVO

AL CRITERIO

CUALITATIVO EN

LA EVALUACIÓN

DE IMPACTO

AMBIENTAL

FROM THE

QUANTITATIVE TO

THE QUALITATIVE

CRITERION IN

ENVIRONMENTAL

IMPACT

ASSESSMENT 
de la autoridad competente. Se plantea que cuando el titular determine, a partir del análisis de características cuantitativas y cualitativas de la modificación de un proyecto, que la misma no requiera ser aprobada por la autoridad ambiental competente, debería realizarse una modificación sin aprobación previa por la autoridad competente, a fin de reflejar la modificación/ampliación de la actividad propuesta en el estudio. La modificación, sin aprobación previa, debería ser informada a la autoridad competente con la finalidad de comunicarle que la actividad ha variado y, por lo tanto, que dicha variación ha sido recogida en el EIA.

La comunicación indicaría que el administrado ha determinado que, de acuerdo con la legislación vigente, no corresponde solicitar la aprobación de la modificación; pero que en aras de una gestión ambiental transparente, y a fin de facilitar los mecanismos de supervisión y fiscalización por parte de las autoridades competentes, cumple con comunicar la modificación poco significativa del proyecto. Si bien tal modificación no requeriría de un pronunciamiento aprobatorio por parte de la autoridad (es decir, una nueva certificación ambiental o la aprobación de la modificación por parte de la autoridad sectorial competente), es importante tomar en consideración lo siguiente: conforme con el artículo 25 de la Ley General del Ambiente, ley 28611, los EIA son instrumentos de gestión que contienen una descripción de la actividad propuesta y de los efectos directos o indirectos previsibles de dicha actividad en el medio ambiente físico y social, a corto y largo plazo, así como la evaluación técnica de los mismos. Deben indicar las medidas necesarias para evitar o reducir el daño a niveles tolerables e incluir un breve resumen para efectos de su publicidad.

El artículo 55 del reglamento de la Ley del SEIA, aprobado por decreto supremo 019-2009-MINAM, señala que «la certificación ambiental obliga al titular a cumplir con todas las obligaciones para prevenir, controlar, mitigar, rehabilitar, compensar y manejar los impactos ambientales señaladas en el Estudio de Impacto Ambiental». Asimismo, los instrumentos de impacto ambiental implican la implementación de medidas de supervisión por parte del Organismo de Evaluación y Fiscalización Ambiental (OEFA). Es decir, implican la verificación de las acciones desarrolladas en el marco de los estudios ambientales aprobados por la autoridad competente en el ámbito del proyecto, de acuerdo con el artículo 75 del reglamento del SEIA.

Si bien el artículo 30 del reglamento de la Ley del SEIA establece que recién al quinto año de iniciada la ejecución del proyecto deberá actualizarse el estudio ambiental en aquellos componentes que lo requieran, debiendo precisarse sus contenidos así como las eventuales modificaciones de los planes que contiene el mismo. La modificación del EIA en plazos distintos al señalado en el artículo 30, aun cuando 
no requiera de su aprobación formal, tiene sentido pues permite al administrado anticiparse a un eventual mandato proveniente de la autoridad competente en el sentido de que corresponde actualizar el EIA, y porque permite prevenir posibles contingencias derivadas de un procedimiento de supervisión y fiscalización.

Al respecto, el artículo 78 del Reglamento del SEIA señala:

Si como resultado de las acciones de supervisión y fiscalización de las obligaciones establecidos en el estudio ambiental aprobado, se determinase que los impactos ambientales negativos generados difieren de manera significativa a los declarados en la documentación que propició la Certificación Ambiental, la autoridad en materia de supervisión, fiscalización y sanción ambiental, que ejercen funciones en el ámbito del SEIA requerirá al titular, la adopción de las medidas correctivas o de manejo ambiental que resulten necesarias para mitigar y controlar sus efectos, sin perjuicio de requerir la actualización del estudio ambiental, ante la autoridad competente, en el plazo y condiciones que indique de acuerdo a la legislación vigente. Esta condición no exceptúa la eventual paralización de operaciones o la aplicación de otras sanciones que pudieran corresponder.

Si bien no se trata de una norma vigente, sino de un proyecto prepublicado por el Ministerio de Energía y Minas, el proyecto de reglamento de Gestión Ambiental para las Actividades de Explotación, Beneficio, Transporte y Almacenamiento Minero (que reemplazaría al decreto supremo 016-93-EM) contempla la comunicación por escrito a la Dirección General de Asuntos Ambientales Mineros, previa al inicio de actividades y obras involucradas, de aquellas modificaciones que no requieran de la tramitación de su aprobación. De acuerdo con este proyecto de reglamento, a la comunicación deberá adjuntarse un informe técnico respaldado por una entidad autorizada para elaborar estudios ambientales.

Por tanto, modificar e informar a las autoridades competentes permite que la OEFA realice la supervisión de las actividades conforme al instrumento ambiental modificado, el mismo que deberá contener todas las modificaciones del proyecto, así como los potenciales nuevos impactos, de ser el caso, y las correspondientes medidas de manejo ambiental.

\section{VI.2. Modificación de la DIA y el EIA-SD}

Hasta el momento únicamente se ha analizado la modificación del EIA propiamente dicho. Ello en virtud de que los reglamentos subsectoriales de minería y electricidad únicamente contemplan la figura del EIA. 
El reglamento ambiental de hidrocarburos contempla reglas para la modificación del PMA, sin precisar si el ámbito de aplicación de estas normas alcanzan tanto a los PMA de los EIA como a los PMA de los EIA-SD, y al «PMA» o capítulo equivalente sobre gestión de impactos de una DIA.

Es por ello que en esta sección se analizarán algunos avances que ha presentado la legislación ambiental para la exploración minera sobre modificación de proyectos y de los correspondientes instrumentos de evaluación ambiental. Legislación que, sin desprenderse de los criterios cuantitativos, integra algunos criterios ambientales que le dan una mayor aproximación cualitativa a la determinación de medidas y parámetros para la modificación de los instrumentos de evaluación ambiental.

Cabe señalar que no se realiza este mismo análisis para actividades eléctricas a las que les es exigible un instrumento de evaluación de impacto ambiental distinto al EIA, porque no existe regulación sectorial que contemple dichos supuestos. En este caso, queda la duda de si le serían aplicables las mismas normas de modificación de EIA por integración analógica, al no existir normas que resulten aplicables a las actividades eléctricas que por sus impactos ambientales califiquen en las categorías I y II. El artículo 33 del reglamento Ambiental para las Actividades de Exploración Minera, aprobado por decreto supremo 020-2008-EM, señala que «El titular podrá modificar el alcance de la DIA aprobada sin exceder los parámetros descritos en el artículo $20^{\circ}$, numeral 20.1. para la Categoría I, debiendo comunicar previamente a la DGAAM y al OSINERGMIN, los cambios a efectuar». Esto quiere decir que puede ampliarse un proyecto de exploración minera clasificado en la categoría I y su DIA puede modificarse, sin necesidad de aprobación previa a la autoridad (únicamente comunicación), si la ampliación del proyecto no origina que el mismo sobrepase la categoría I de evaluación de impacto ambiental para actividades de exploración minera.

Como quiera que la determinación de categorías para la evaluación de impacto ambiental en el marco del reglamento de Protección Ambiental para Actividades de Exploración Minera se basa netamente en un criterio cuantitativo, la modificación de la DIA parte de ese mismo criterio. Es decir, si la modificación del proyecto hace que el proyecto en su conjunto tenga un máximo de veinte plataformas, el área efectivamente disturbada es menor a diez hectáreas y la construcción de túneles es hasta de cincuenta metros en su conjunto, entonces la modificación de la DIA no tendría que ser previamente aprobado por la autoridad. Bastará la comunicación previa a las autoridades competentes de su aprobación y fiscalización sobre los cambios a efectuar. Cabe resaltar, sin embargo, que si bien este reglamento se sustenta básicamente en un criterio cuantitativo para determinar si la modificación de una 
DIA requiere de aprobación previa de la autoridad, también es cierto que se basa en criterios ambientales para exigir una modificación con aprobación previa por parte de la autoridad sectorial.

Acertadamente, se exige la modificación de la DIA con aprobación previa de la autoridad ambienta sectorial cuando la modificación al proyecto se vaya a ubicar a menos de cincuenta metros de un bofedal, canal de conducción, pozos de captación de aguas subterráneas, manantiales o puquiales; en glaciares o a menos de cien metros del borde del glaciar; en bosques en tierras de protección y bosques primarios; y en áreas que tengan pasivos ambientales mineros o labores de exploración previas no rehabilitadas, que excedan el nivel de intervención que configura la categoría I. Asimismo, indica que de excederse los parámetros que califican a un proyecto dentro de la categoría I, deberá iniciarse un procedimiento de aprobación de un EIA-SD. En dicho caso, los criterios para determinar si una actividad debe mantenerse en categoría I o si le corresponde ser calificado dentro de la categoría II y corresponderle un EIA-SD serán, como hemos visto, netamente cuantitativos.

En el caso de los EIA-SD, el reglamento exige que toda modificación a los componentes principales del proyecto minero, es decir, a las plataformas de perforación, las galerías subterráneas, trincheras, el área de disposición final de residuos sólidos (trincheras o celdas de seguridad), campamentos permanentes, el área de almacenamiento principal de combustibles, el área de almacenamiento de desmontes, área de almacenamiento de mineral, infraestructura para el manejo o tratamiento de aguas y los accesos previamente sea aprobada por la autoridad ambiental competente. No requerirán aprobación previa las modificaciones auxiliares que no se encuentren, como en caso de la categoría I, a menos de cincuenta metros de un bofedal, canal de conducción, pozos de captación de aguas subterráneas, manantiales o puquiales; en glaciares o a menos de cien metros del borde del glaciar; en bosques en tierras de protección y bosques primarios; y en áreas que tengan pasivos ambientales mineros o labores de exploración previas no rehabilitadas. Estas últimas también requerirían de aprobación previa por parte de la autoridad ambiental competente.

Estos criterios ambientales comprueban que el reglamento para Actividades de Exploración Minera, por ser un reglamento más moderno, en vigencia desde el año 2008, intenta incluir variables ambientales en el análisis tanto de la modificación de los instrumentos de evaluación ambiental, como de su categorización. Vemos pues que dentro de parámetros cuantitativos referidos al número de plataformas, longitud de los túneles, o magnitud del área afectada introduce algunos elementos cualitativos relacionados a áreas vulnerables en las que podría ocasionarse per se una afectación al medio ambiente.

DEL CRITERIO CUANTITATIVO AL CRITERIO CUALITATIVO EN LA EVALUACIÓN DE IMPACTO AMBIENTAL

FROM THE QuANTITATIVE TO THE QUALITATIVE CRITERION IN ENVIRONMENTAL IMPACT ASSESSMENT 


\section{CONCLUSIONES}

El Sistema Nacional de Evaluación de Impacto Ambiental, regulado tanto por la ley como por el reglamento de la Ley del SEIA, ha mostrado un avance significativo en materia de certificación ambiental, estableciendo parámetros más coherentes en defensa del medio ambiente. En lo que a modificación de estudios aprobados se refiere y su aprobación por la autoridad competente, este avance consiste fundamentalmente en haber abandonado rígidos criterios cuantitativos, basados en la envergadura de un proyecto, hacia criterios cualitativos más acertados, enfocados en los efectos particulares sobre el medio ambiente de cada modificación de los proyectos previamente certificados ambientalmente. En ese sentido, la ley y el reglamento del SEIA dan un giro en la concepción sectorial de la certificación ambiental, poniendo el acento en el lugar correcto, en la identificación, minimización y manejo de los potenciales impactos ambientales de la actividad humana sobre el medio ambiente.

Sin embargo, como se ha analizado en este artículo, tanto la ley como el reglamento dejan cabos sueltos en la aplicación de los criterios ambientales, específicamente en materia de modificación de los instrumentos de evaluación de impacto ambiental, al estar regulado por cada uno de los reglamentos de protección ambiental sectorial, poco o nada se tienen en cuenta los criterios ambientales de afectación del medio ambiente, utilizándose criterios rígidos que toman en consideración el tamaño o ampliación del proyecto. En algunos subsectores y para algunas actividades, esta deficiencia se observa no solo al momento de determinar la obligatoriedad de la aprobación de la modificación del estudio ambiental, sino en la clasificación de las propias actividades para determinar el tipo de estudio que les corresponde. En ese sentido, este artículo intenta ser un llamado de atención para romper las ataduras al paradigma cuantitativo, en función de la envergadura del proyecto, y priorizar el paradigma cualitativo en función de los impactos y la urgente protección adecuada al medio ambiente. 